\title{
Affective Processes as Network Hubs
}

\author{
David Gibson \\ Curtin University \\ david.c.gibson@curtin.edu.au
}

\begin{abstract}
The practical problems of designing and coding a web-based flight simulator for teachers has led to a 'three-tier plus environment' model (COVE model) for a software agent's cognition (C), psychological (O), physical (V) processes and responses to tasks and interpersonal relationships within a learning environment $(\mathrm{E})$. The purpose of this article is to introduce how some of the COVE model layers represent preconscious processing hubs in an AI humanagent's representation of learning in a serious game, and how an application of the Five Factor Model of psychology in the O layer determines the scope of dimensions for a practical computational model of affective processes. The article illustrates the model with the classroom-learning context of the simSchool application (www.simschool.org); presents details of the COVE model of an agent's reactions to academic tasks; discusses the theoretical foundations; and outlines the research-based real world impacts from external validation studies as well as new testable hypotheses of simSchool.
\end{abstract}

Keywords: affective processing models, cognitive models, Five Factor Model of psychology, teacher education, game-based learning, simulation

\section{Affective Processes in a Serious Game for Training Teachers}

A web-based training simulation - simSchool - has been built to improve teacher education because what teachers do in the classroom matters a great deal and is part of a causal network that brings about student learning as evidenced in the teacher's skill- and knowledge-based performances [1]-[3]. Teacher decisions can be thought of as independent variables in an ongoing experiment that builds expertise over time $[4,5]$. A major challenge facing beginning as well as experienced teachers is how to juggle a number of parameters in an often-overwhelming complex setting where learner psychological characteristics, cultural and family backgrounds, content requirements, school routines, state and national policies, and community expectations intersect. For example, teachers must learn to constantly negotiate a balance between content, pedagogy, and technology in ways that are appropriate to the specific parameters of their content area and an ever-changing educational context [6]. They must learn to differentiate instruction to meet the needs of mixed ability groups [7]. And 
they must develop the ability to self-assess and plan for their own professional growth while encountering a variety of pedagogical approaches in teacher education [8].

Normally, these capacities are developed through a combination of teacher education experiences followed by student teaching in real classrooms and then honed further during their first job as a schoolteacher and subsequent years on the job. However, two major problems with this situation are evident; 1. Governments experience low retention rates from teacher preparation to lifelong teaching and 2. There are ill impacts of inexperienced teachers on student learning that are known to harm some students and prevent many of them from reaching their potential.

The possibility of using a simulation to help develop and assess the performance of teachers has arisen in concert with a growing appreciation of the potential for games and simulation-based learning [9]-[11]. Using simulations in teacher education has at least two broad goals; producing better teachers and building operational models of physical, affective processing, cognitive, social and organizational theories involved in teaching and learning [12], to which we will turn in the following sections. The broad goals are situated in the context of using technology to improve field experiences for preservice teachers and in ongoing professional development of inservice teachers, where simulations can provide learning and training opportunities with unique characteristics such as repeatability, automated analysis, representations that spark reflection, and the potential to transfer lessons learned and higher teaching skill levels to the real classroom [13]. In addition, the significance of using a digital simulation as an assessment environment includes more authentic item and response types, scalability, safety and usability for formative assessment and learning, and production of rich data; and when combined with an evidence-centered assessment, can provide high-resolution performance data linked to a standards-based model [14, 15].

In this context, simSchool was created to develop capacities needed for teaching and to also address the major problems of field-based experience, reasoning that a scalable simulation would have far fewer ill impacts on real students while a candidate was developing skills and the confidence to become an effective teacher. Previous research has indicated that teacher development via simSchool does indeed lead to increased self-efficacy, improved teaching skills and a positive shift in the locus of control of classroom learning in preservice teachers $[16,17]$. In addition, a recent study indicates that exposure may also impact teacher retention [18].

It is important to point out at the outset that the computational model underlying simSchool described here treats affective states as a relatively undifferentiated continuum from unexpressed, sometimes inexpressible, states that might transform into recognized and relatively persistent states of mind. This contrasts with affective states seen as a small number of expressible states such as grief, love, frustration, boredom, and joy. The affective continuum stance is supported by both cognitive disequilibrium theory $[19,20]$ which dynamically links affect and cognition during learning and the requirement of a fine-grained analysis of the rapid dynamics of processes that naturally occur during learning, for modeling cognitive-affective dynamics [21]. The finegrained preconscious continuum model may not be appropriate for understanding sustained affective states, or the end points of continua of affect (e.g. love, anger) that have become conscious, but it is critical to negotiating the dynamic change during a 
learner's experience, for example a learner's state of engagement with a task or a conversation changing to confusion then to frustration during a learning process.

A second important issue of the model that requires additional introductory explanation is the foundation of the fine-grained continuum in the Five Factor Model or OCEAN model of psychology [22]. The OCEAN model is typically used as a 'trait' theory [23], but here it is used as scope for the dimensionality of a large but finite number of 'states' of preconscious processes (e.g. affects and emotions if made conscious), so additional explanation is needed. State-trait distinctions can be analyzed as having four overlapping but distinct dimensions: duration, continuous vs reactive manifestation, concreteness vs abstractness and situational vs personal causality [24].

To illustrate, consider the face validity of a learner who is facing a performance challenge, such as singing one's national anthem in front of an audience. Even if the person is normally 'open' to new ideas (e.g. the 'O' in the OCEAN theory), the singing task calls upon memory of words, remembering a melody and the production of sound in a musical context, in front of an audience. So the person will need psychological and mental resources related to the repetition of familiar tasks, which will cause disequilibrium and send the person through a range of preconscious states to deal with the gap between a current state and the one needed to perform the required task. Using the four state-trait distinctions in this example, the song will be over soon (duration) as opposed to a trait set point of openness, which remains after the song is over; the disequilibrium will change to equilibrium when the performance requirement is removed or the performance concludes (reactive manifestation returning to continuous manifestation); the context will shift during and after the performance as the audience turns attention to something else other than the person (moving from concreteness on self to abstractness of the group, from the person's perspective); and the personal causal role will subside back into the general contextual situation. It seems natural to infer that a state interpretation of variables operating dynamically over time within the five continua of OCEAN dimensions is feasible for illustrating the change in state from the condition prior to the challenge to sing, to the states experienced during the performance, and those experienced afterwards, when the person returns to a resting state that is more trait-like.

The theory outlined below and used to drive the AI of the simSchool application asserts that a person's current state settings on variables of openness, conscientiousness, extraversion, agreeableness and neuroticism (OCEAN) will shift positions many times before, during and after a required performance. Accompanying these positions will be preconscious passages of partial or fuzzy affective states with a large number of barely differentiated positions within those dimensions driving and being driven by interpersonal interactions [25] as well as driving and being driven by key physical processes [26]. The model has been designed for learning, task performance and interpersonal interaction contexts and may not be valuable or practical for other contexts. 


\section{The simSchool Simulation}

In order to simulate learning, simSchool had to exhibit physical, psychological and cognitive features of classroom learning and allow a user to exercise and test a variety of learning theories. The broad interaction rules thus embody several well-known educational mechanisms including the zone of proximal development [27], mastery and performance goals [28], multiple intelligences [29], differentiation of instruction [7], and culturally responsive teaching [30]. For the focus of this paper, we concentrate on the physical, psychological and cognitive features of an individual learner, which will set the stage to discuss how pre-conscious processing reactions impact the AI representation of the ability to learn. The model of individual learning in simSchool contains ten variables or factors that are organized into three bundles or hubs of physical (visual, auditory, kinesthetic) psychological (openness, conscientiouness, extraversion, agreeableness, neuroticism) and cognitive variables (academic performance capability, and language capability).

A simplifying assumption of the model is that the variables are independent of each other in their responsiveness to the external environment and are grouped together for the display of behavior. So for example, the visual component of a task only impacts the visual capability of the learner (not capabilities in hearing, or thinking or feeling) but the overall affective state computed from the combined impact of all variables leads to a behavior based on all the physical variables as a bundle.

Three hubs (physical, psychological, cognitive) comprised of ten variables hold a past and current state for each individual learner in the simulated classroom. Hillclimbing algorithms (e.g. computing the distance from a goal state to a current state and then reducing that distance) take time to raise or lower the ten variables simultaneously and in relation to the environment (classroom tasks given by the user and conversational interactions). The environment drives the evolution of the variables and the distance-reducing algorithm gives rise to momentary and transitory affective psychological stances of the agents. Those stances, in conjunction with the physical and cognitive challenges of classroom learning, impact the agent's behavior and academic performance.

Together the (V) visceral physical factors, (O) OCEAN-based Five Factor psychological factors and (C) cognitive factors are thus used to represent salient elements of individual emotional response to the (E) environment of classroom learning challenges, in the COVE model [31]. Aspiring teachers interact with simSchool over several sessions, which often take place over several weeks, with microteaching interactions lasting from 10 to 30 minutes. The teacher candidates attempt to negotiate the simulated classroom environment while adapting their teaching to the diversity of students they face.

With this brief background and context, the next section discusses the theoretical framework that guided the design decisions for the model of preconscious processes, including both physical and affective states, in the COVE agent model. 


\section{Design Decisions}

The COVE model treats emotions and other affective states as arising from hubs of regulatory networks of non-cognitive as well as cognitive processing. These networks specifically span from unconscious to conscious processing in order to fulfill a causative and evolving role in shaping values that lead to behavior. Affect and emotions are viewed as participating in an interstitial space between preconscious and fully conscious processing in order to provide a dynamic bridge as well as a structure for the orchestration of subconscious processes to arise into recognizable conscious states which become tagged with emotion and affect terms such as anger, fear and joy. The model differs from the notion of affective processes as "valenced reactions to events, agents or objects" [32] and in particular subjective-based appraisal theory [33] because it does not contain the concept of conscious personal significance. Instead, it uses a metric distance between task requirements and the current state of a person's COVE variables to represent a 'multidimensional gap closing' as the primary mechanism of transitory as well as sustained preconscious and conscious states. The model combines undifferentiated precursor states arising prior to as well as simultaneously with observable reactions and behavior and makes operational a complex landscape of flexible networks of processing centers that give rise via combinatorial interactions to a great number of physical, pre-conscious and conscious states (e.g. approximately $10^{\wedge} 20$ states).

\subsection{Multidimensional \& Multileveled Homeostasis}

Considered during the design decisions of the COVE model, emotion has most often been defined as an affective state of consciousness, distinct from volitional (deciding and committing) and cognition (acquiring knowledge and understanding through thought, experience, and the senses) [34]. In this definition, feeling (emotion), willing (volition), and knowing (cognition) are distinguished from one another as separate aspects of reality, all of which require consciousness. However, in other literature the emotions are also generally understood as representing a synthesis of subjective experience, expressive behavior, and subconscious neurochemical activity. It is this synthesis of physiology with experience and adaptive behavior in particular that opens the door to unconscious and preconscious activity-shaping precursor affective states leading to emotions. For example the homeostasis of underlying physiological processes gives rise to cyclic regimes that alternate between promoting and inhibiting particular emotional responses [35]. This leads to a design decision for the computational model of affective processes to include a multidimensional and multileveled homeostatic relationship with the external world - the agent emotes in a complex adaptive relationship to its context, which is not limited to rational thought or cognitively prescient appraisal. 


\subsection{Balance of Control and Flexibility}

Emotions arose in evolution in order to provide a superordinate coordinating system for potentially competing preconscious subprograms of the mind [27, 28]. An animal could not have survived, for example, if simultaneous cues for sleep and escaping an approaching lion had sent ambiguous signals; emotional reactions help sort things out. Emotions play a shaping role in focusing attention, increasing the prominence of memories, affecting cognitive style and performance, and influencing judgments [12]. As a result of these shaping forces, emotions also play a part in personality development; and of equal importance, one's personality shapes one's emotional life. This aspect is particularly important in relation to learning and classroom performance. This leads to a design decision for the computational model to have the capability of controlling subsystems while maintaining a flexible, adaptive capability.

\subsection{Experience-based Learning}

Emotions have been properly viewed as a form of intelligence involved in learning [38]. In fact, a common ground between non-cognitive (e.g. physical, emotional, psychological) and cognitive (thinking, rationalizing) forms of intelligence is learning. Whether it is the adaptive physical and behavioral structures arising in a survival context, or how linked emotional and behavioral repertoires of a male and female of a species ensures courtship followed by reproductive and parental roles, or how a flushed face projects an internal state to others in a social setting, something has been learned via evolution if not during one's lifetime. This leads to a design decision for the computational model to be able to learn from experience, allowing the development and expression of intelligence.

\subsection{Affective Processes as Pre-Conscious Processes}

The literature on emotional intelligence has for the most part focused on conscious processes that are seen as syndromes of conscious thoughts, feelings and actions [39]. This may be due to the fact that the study of human intelligence is in general, reliant upon psychometric methods that observe or poll people behaving in a social or testing setting. However, alternative theories of intelligence assert that consciousness is not required, for example the intelligence of lower forms of life [40], artificial life [41], the intelligence that is distributed across both the world and evolutionarily determined bodies [42], and the group intelligence of social animals that individually lack consciousness [43]. This evidence influenced the design decision to treat affective processes as primarily pre-conscious and secondarily as conscious processes.

\subsection{Time-based Learning}

Since learning needed to be explicitly integrated into the simSchool model of agent emotions, the COVE model includes the layered characteristics of human learning. Typologies have arisen to describe those layers to account for a wide variety of learn- 
ers in terms of physiological and psychological factors, and to explain how differing forms of intelligence arise in cultures and communities of practice [35, 36]. Integrated with these conceptions and refining them, layered approaches to cognition have been discussed by neuroscientists [37, 38], psychometricians [48], cognitive scientists [49] and computer scientists [32, 41, 42]. For example, Bruner [49] discussed cognitive development using a three-stratum framework of "enactive, iconic and symbolic" and Carroll's [52] factor-analytic model defined three layers as "narrow, broad, and general." These layered models have in common the idea that learning progresses from specifics negotiated at a "lowest level" active layer interfacing with the environment, to generalities synthesized from abstractions at higher levels. This leads to a design decision that enables the model to develop over time both in evolutionary timescales and during an individual's lifetime through a layered process of learning.

\subsection{Design Decisions Summary}

These design considerations set the stage for a computational model of affective processes that (1) traverses and operates across several levels: from physical, through pre-conscious processing layers, to higher cognitive areas that are capable of labeling experience; (2) maintains a critical balance between flexibility and control; (3) maintains a homeostatic dynamic equilibrium with both internal and external world features; and (4) learns from experience.

\section{The COVE model}

COVE uses three layers to organize the internal variables of an AI student in simSchool and one layer to represent the external context of learning (Figures 1 and 2). The E layer is the external environment, which in a classroom includes the task set before a student by the teacher and things the teacher might say to the student, as well as what others in the class say and do in reaction to the same stimuli. The V layer is the visceral, early-stage physical and pre-emotional processing stratum. The O layer contains the emotions and other pre-conscious processing, and the $\mathrm{C}$ layer contains conscious processing. The full COVE model uses a modified psychometric approach based on the factor-analytic model (sixteen factors) proposed by Cattell-Horn-Carroll (the $\mathrm{CHC}$ theory of intelligence), which has been validated and is widely used to understand cognitive abilities. simSchool uses ten of the sixteen factors and also uses a blend of structural-functional learning theories, which are needed to fully model the holistic context of a classroom learning context.

For each factor, the model adopts a representation as either a bipolar continuum of qualitatively different capabilities or a combination of a threshold with a qualitative continuum. For example, the skill of mathematical computation can be represented on a continuum with low positions on the scale representing basic arithmetic skills and high positions representing abstract or symbolic computations of higher orders. The number of positions on each continuum is selected to balance computational flexibility with representational accuracy (e.g. typically from five to twenty). The choice of 
number of levels and factors increases the computational possibilities and challenges for modeling. A fully connected 16 factor cognitive model with 5 levels on each factor, for example would have $5^{\wedge} 16$ connection possibilities.
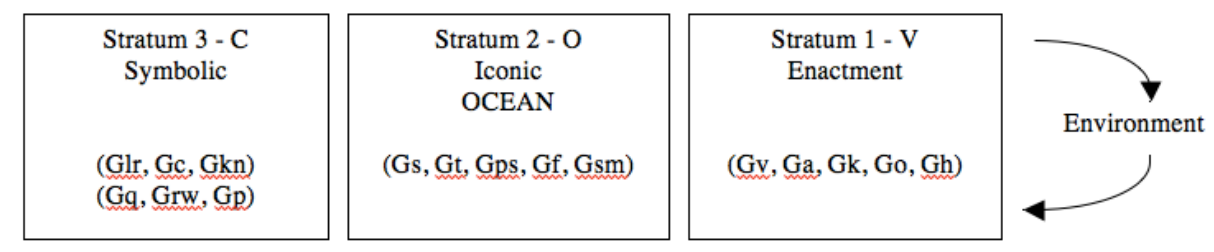

Fig. 1. COVE model of cognition integrating the $\mathrm{CHC}$ theory of intelligence

Evidence for simplifying the number of relationships through layering and hierarchical networks is available from intercorrelation data among the broad factors [53]. For example, for people aged 14-19 who took part in the development and standardization of the Woodcock-Johnson III, comprehensive knowledge (Gc) was .62 correlated with fluid reasoning (Gf) but only .37 with processing speed (Gs). This suggests that there may be a closer relationship between Gc and Gf. In addition, structural and functional considerations suggest a narrowing and channeling of the factors. For example, perception usually precedes cognition and the consolidation of long-term memory is facilitated by emotional arousal [54] implying that the layers handling perception $(\mathrm{V})$ must link with emotional and psychological layers $(\mathrm{O})$ before linking with long-term memory and crystallized knowledge (C). These kinds of considerations lead to a layered model (Figure 2) consistent with Hawkin's ideas about hierarchical and time-based memory that is stored as sparse dynamic patterns changing over time [55].

\subsection{The C Layer}

The "C" layer of the COVE model utilizes 6 of the 16 CHC factors to model conceptual knowledge (Figure 1): general storage and retrieval (Gc, Glr, Gkn); and specific storage and retrieval abilities (Gq, Grw, Gp). In the simSchool application, two of these six are directly represented in explicit variables (Gc and Gkn). Processes and relations in the algorithms function as the remaining factors. As higher cognitive functions, Gc is used to represent generalized academic capability and a specific type of Gkn (language) represents the degree to which the simulated student's language is the majority language of the classroom.

\subsection{The O Layer}

The "O" layer of the COVE model utilizes $\mathrm{CHC}$ factors involved in processing and reasoning (Gf, Gs, Gt, Gps, Gsm). The "O" layer is an interface between intelligence and personality in which one's psychological make-up is dominant and involved in basic central information processing mediated by emotions. In addition, the psycho- 
logical model - that we assert is the crucible of affect and emotions - utilizes the Five-Factor (or OCEAN) Model of psychology [56] as the foundation of the student personality spectrum. The factors are treated as states (not traits) to allow evolution over short time spans during a single class session. Theoretical justification for the state-trait distinction was introduced above. For each of the five variables a continuum from negative one to positive one (increments of .1) is used to situate the learner's specific pre-conscious processing propensities, which shift as the context of the classroom changes.

Following Ortony, Clore, and Collins [32] but extending into subconscious processes, the "O" layer assumes that reactions developing during the appraisal of a situation influence performance. Appraisal in the COVE model can be physical (e.g. hair standing up on the back of the neck), subconscious (e.g. a smell and time of day triggering a nondiscursive association), conscious (e.g. a feeling clearly associated with a linguistic label such as shame, fear or attraction), or any combination. With continuous factor subcomponents, the COVE model treats affective states as a large number and wide range of states from preconscious to conscious that result from factor interactions as the variable settings on OCEAN ascend and descend around their set points; similar in spirit to Russell's continuous two-factor model [57].

Individual psychology is represented in COVE by the "Five Factor Model of Personality," "Big Five," or OCEAN model [58], [59]. OCEAN stands for Openness, Conscientiousness, Extroversion, Agreeableness and Neuroticism. Each factor has an opposite (e.g. the opposite of Extroversion is Intraversion). The OCEAN taxonomy encompasses several important psychological characteristics of learners and is represented by a continuum on each factor. The end of each continuum has maximum value in a variable or its opposite. The link of OCEAN to affect is established, for example by well-known affective disorders that are known to have OCEAN model correlates $[60,61]$ with certain maxima and minima.

In simSchool (www.simschool.org), the OCEAN variables are set on a scale from -1 to 1 , with 0 at the midpoint, which allows the software agent to possess a $21^{\wedge} 5 \mathrm{mix}$ of valences upon which to base its observable behavior. SimSchool divides the scale into .1 units, giving 21 positions from -1 to 1 (e.g. $-1,-.9,-.8 \ldots .8, .9,1$ ). This provides the "O" layer of the agent-learning model with over 4 million OCEAN-related states. The application narrows the observable descriptions of these possibilities by grouping the factors into 5-position narratives, representing clusters near $-1,-.5,0, .5$ and 1 . This creates a space of $5^{\wedge} 5$ or 3125 narrative descriptions that act as clues about the underlying 4 million actual states, in "student records" that a user can read in the role of playing the teacher of a simSchool class. The narratives are dynamically assembled from a database to create each unique personality and are presented to the user on demand as well as during the course of a simulation. Affective states (a concept that is proposed to apply to some of the 4 million OCEAN-based states) affect the overall behavior of the agent as one of the three hubs of data in the current state space of the agent, which is then mapped to a much smaller behavior space.

Linking OCEAN to the CHC model of intelligence (Figure 1 and 2) has been proposed based on correlation evidence from studies of subjectively assessed intelligence (SAI) [62] . An example of SAI is a student who has often failed tests, which leads 
the student to an expectation to fail a future test, and thus lowered performance on the future test influenced by his or her appraisal. Citing a number of studies, it has been proposed that SAI mediates between personality, intelligence and performance, with a number of correlations noted by researchers, including:

- Personality traits are significant predictors of academic achievement

- (Gf) and (Gc) are both positively correlated with performance

- Openness $(\mathrm{O})$ is positively correlated with intelligence

- Conscientiousness (C) is a positive predictor of performance

- Extraversion (E) is positively correlated with intelligence which is assumed to be due to higher speed of response (Gs, Gt, Gps) and lower arousal (N-)

- Neuroticism $(\mathrm{N})$ is a negative predictor

The COVE model links OCEAN to $\mathrm{CHC}$ at the "O" layer reasoning that OCEAN is more complex than receptor-based perception at layer " $\mathrm{V}$ ", and more immediate but less complex than conceptualization and long term memory at layer "C." In addition, following Eysenck and Eysenck [26] who suggested that SAI should be considered a part of personality rather than intelligence and Chamorro-Premuzic and Furnham [62] who note the "considerable conceptual overlap between the concept of SAI and Openness" (p. 256), the COVE model layer "O" situates psychology, emotions, and reasoning fluidity (Gf) to fulfill the non-cognitive appraisal function.

The correlation evidence and structural-functional considerations lead to a model of "O" that includes causal precedence in the incoming signals from the environment (Figure 2). Intercorrelation of Neuroticism and Extroversion with Openness, Conscientiousness and Agreeableness is suggested based on neurophysiological evidence from animal and human studies that posits two large clusters: (1) Extraversion, Exploration, Novelty seeking, Sensation Seeking, Positive Affectivity and Impulsiveness, versus (2) Neuroticism, Anxiety, Fearfulness and Negative Affectivity [24]. The two large $\mathrm{E} \& \mathrm{~N}$ clusters are mediated by independent neurobiological mechanisms (e.g. catecholamines, dopamine and norepinephrine for $\mathrm{E}$; and the amygdala and the benzodiazepine / GABA receptor system for N). The arrows in Figure 2 all represent positive correlations.

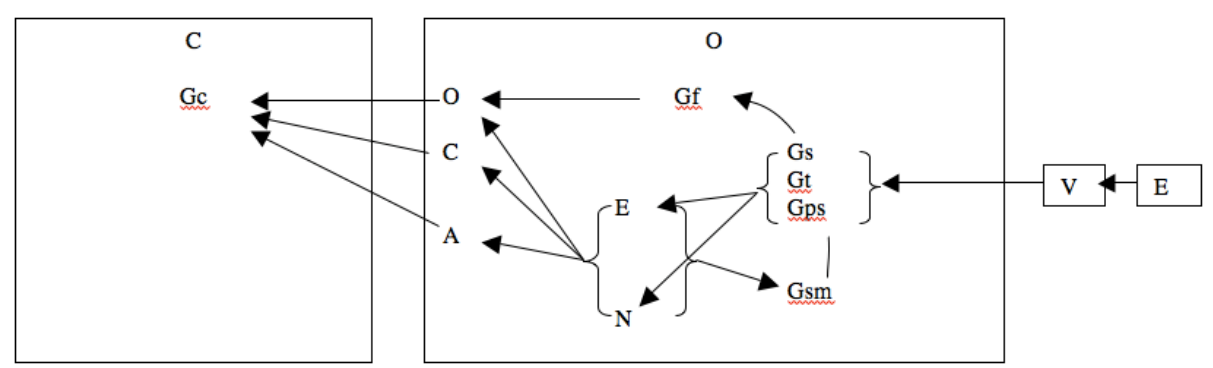

Fig. 2. Linking CHC to OCEAN variables 
The COVE model is an attempt to describe the contents and the mechanisms of environmental responsiveness, information processes, affect, emotions and thought, but much work remains to be done. For example, the pathways in Figure 2 focus on the "incoming" signals leading to crystallized knowledge; however, returning pathways from pattern formation, recognition, beliefs, and decisions to intentions and action exist at every level too. The simSchool application creates a simple mapping of current state to the mechanisms that update the state over time and the externally supplied goal of responding to the environment. Appraisal of a situation is an unconscious process that in simSchool takes place in eight of the ten dimensions ( $3 \mathrm{~V}$-layer, 5 O-layer) and results in behaviors that become visible to the player. The simSchool model of today narrows the focus of appraisal to that of learning-task performance (objects in the environment), teacher conversations (agents in the environment) and the evolution of both of those influences in sequences (internal as well as external events in all layers).

The arrow in Figure 2 from the $\mathrm{E}$ to $\mathrm{N}$ subcomponent sends a reentrant signal within the O layer via short term memory (Gsm) to the (Gs, Gt, Gps) cluster dealing with processing and leading to flexibility (Gf) and Openness. This mapping allows for reinforcement learning as well as cyclic reappraisal [63]. In addition, with the returning pathways that provide internal situational content [64], the mechanism described by Lahnstein [65] can be supported, where the onset and decay of an emotive episode is shaped by dynamics of interactions with previous states. Finally, the reentrant loop also introduces time and time delay into the mapping, without which Figure 2 would be a primarily feed-forward network.

\subsection{The V Layer}

The "V" layer of the COVE model includes the five factors related to sensory perception $(\mathrm{Gv}, \mathrm{Ga}, \mathrm{Gk}, \mathrm{Go}, \mathrm{Gh})$. The physiological characteristics involved in learning entail both sensory (afferent) and motor (efferent) neural pathways. While learning is sometimes thought of as primarily the organization of incoming sensory signals, recent work in artificial intelligence and robotics as well as constructivist learning theories suggests that pre-motor and motor systems - the body's exploration and action in the world - plays a major role in the development of intelligence [42]. The "V" layer concentrates on the sensory components of learning. In the simSchool engine, only ( $\mathrm{Gv}, \mathrm{Ga}, \mathrm{Gk})$ are used since those are more typical in classroom learning.

In these physiological or " $\mathrm{V}$ " variables, unlike the bipolar "O" psychological variables, there is the possibility of a complete absence of an input pathway, such as in blindness or deafness, thus the use of a threshold level in addition to a range of ability or preference. The physical sensory model thus utilizes a quasi-continuous scale from zero to one (increments of .1) to represent the simulated student's strength and preference (e.g. a setting of zero means that the simulated student cannot see and has no preference for visual information; a setting of one indicates that the student can both see and has the highest possible preference for visual information). 


\subsection{The E Layer}

The "E" layer of environment variables in the COVE model includes learning tasks that involve the nature of knowledge (objects), interpersonal relationships and expectations states theory [66], [67] (agents) and the effects of sequences of interactions (events). In a recent review of learning theory [68], environment also includes "community," which reflects the social context of learning and the feedback role of external "assessment." In addition, some aspects of the nature of knowledge itself are external to the individual learner, namely objective reality. The COVE model is thus evolving to contextualize cognition as a social, cultural, and psychological interaction of internal and external factors, not solely as an "information processing" or "knowledge acquisition" problem of an individual.

In simSchool, signals from the environment are of two types: tasks have duration and influence continuously, conversations are point-like events with a pulse of influence that decays over time. The user can control which tasks are impacting which simulated students, so a high degree of differentiation is possible. Instances of talking are likewise controllable to impact a single student or the group as a whole.

\section{$5 \quad$ Agent Learning Process}

At its simplest, the process of learning in the model is represented by the complete acquisition of each of the ten variable targets set by any task assigned or talking behavior of the user. The process takes variable amounts of time because of the role of the $\mathrm{O}$ layer emotional reactions within the rest of the complex of other variables. In practice, the targets are almost never fully attained because either time runs out when the user changes the task or quits the simulation, or the simulated student begins to act out because a threshold level of task completion is reached, slowing the pace of additional progress. In simSchool, the appearance of learning is knowable via the performance of the agent in relation to the characteristics of a task, as the task attractors (or repellors) for the ten variables in the COVE model of each simulated student shapes each performance. Affects are envisioned as the transitory and momentary states passed through as the agent integrates the environmental triggers of the tasks and talking with their current internal state. The ten-dimensional model provides numerous states with specific implications for externally observable behaviors; for example, a student who is highly extraverted when given a task that is highly introverted, has a clear trajectory on that variable, while another student may be highly introverted and will have almost none. The learning process and any related affective states for each of these agents will be different. These differences give rise to roughly $20^{\wedge} 10$ internal states (as opposed to 6 to 8 labeled affective states) in the possibility space for agent behavior. 


\section{$6 \quad$ Validation Studies}

No formal studies have yet validated the computational modeling of either subjective affective states or the larger field of preconscious states traversed by a learner during a process of learning. However, internal model validation of simSchool has been achieved in incremental stages using expert and literature review, and controlled testing with the platform. To facilitate validation by any user, visualizations of the transitions of the ten variables in the model have been built as part of the user interface, so that all the actions of the variables are transparent, allowing a user, for example, to hold nine variables constant while testing for the directionality of a tenth test variable. The magnitude and speed of changes have not been verified in the model at this stage of development, but the directionality of all variables has been verified by experiment and can be replicated by any user.

For example, consider the expected direction of variables in the user described earlier who needs to sing the national anthem in front of an audience. If the person is highly 'open' (distractible) and 'neurotic' (fearful) and the singing task requires a high degree of 'repetition from memory' (focus) and 'steeled nerves to face an audience' (emotional stability) compared to a completely similar AI person but with less openness and less neuroticism, then the result will show a noticeable difference in the time and shape of the variable trajectories needed for successful performance. A person with the requisite openness and lack of neuroticism will succeed at the task quicker than otherwise, and the trajectories of all variables will be noticeably different via visual inspection (Figure 3) giving insight into the model's causal explanation of the dynamics. Remembering the theoretical foundation of cognitive dissonance [20] and the need for high resolution rendering of affective and cognitive transitions [21], the visual inspection method of validation has succeeded in supporting judgments of the model's validity, which has also later been shown to be effective in impacting a user's intuition and judgments in externally validated contexts described next.

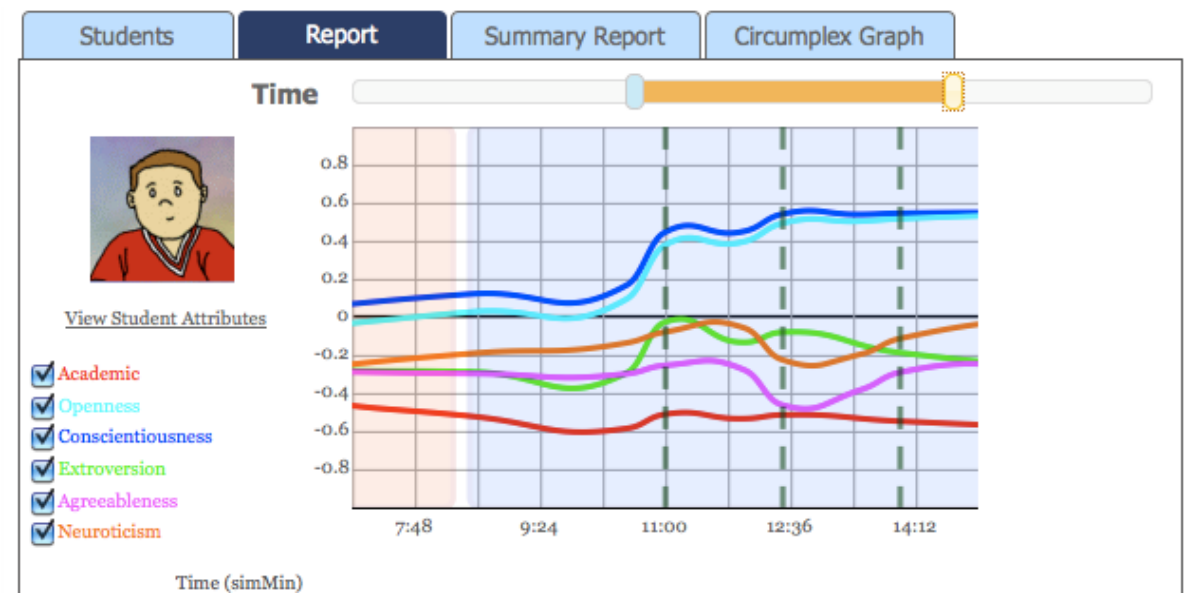

Fig 3. Trajectories of OCEAN variables during a task in simSchoo 
Since the mapping of a dynamic set of internal mental states (e.g. physical, psychological and cognitive) to observable behavior (e.g. student records, grades, sitting, speaking and evidencing academic progress) in a classroom context is the whole point of the COVE model and the model was created in order to stimulate a user's insight into teaching and learning processes, several external validation studies have been conducted on the real world impacts of the simulation in its intended use context. These studies indicate that the COVE model adequately represents classroom learning differences [69], [70], and facilitates positive changes in teacher confidence, skills, and attitudes [16], [71].

One study used the Teacher Preparation Survey (TPS) a 25-item, Likert-based instrument divided into two sections, one about perceptions of teaching situations, and the other about teaching skills. It was adapted from [72]. The instrument was found to have both content validity as well as construct validity as determined through factor analysis [16]. In 2007 the instrument was found to have acceptable internal consistency reliability (Cronbach's Alpha) for Instructional Self-Efficacy (Alpha $=.72$ ) but not as high for Learning Locus of Control (Alpha $=.57$ ). Post hoc internal consistency reliability analysis for one 15-item factor produced a Cronbach's Alpha value of .97. These pedagogical scales were reconfirmed on an additional set of data. The 25 items from the TPS were resubmitted to a single exploratory factor analysis (Principal Components, Varimax rotation). The three-factor solution converged in four iterations and all items loaded on the anticipated factors. Cronbach's Alpha values for these scales were: Instructional Self-Efficacy $=.77$ (5 items); Learning Locus of Control = .68 (5 items); and Teaching Skill $=.95$ (15 items). Internal consistency reliability estimates were all in the range of "acceptable" to "very good" according to standard guidelines [73]. Items composing Teaching Skills, Instructional Self-Efficacy, and Learning Locus of Control scales are listed in an earlier publication featuring development of these indices [71].

Two studies in subsequent years found similar results, leading the research team to conclude that pre-post data gathered at three points in time across five years indicates that simSchool in a preservice teacher candidate environment measurably increases Instructional Self-Efficacy (confidence in one's competence), Learner Locus of Control, and Self-Reported Teaching Skills [74].

These studies show that the COVE model's map of the preconscious processes to classroom behavior and academic performance are adequate for training teachers and conducting practical educational psychology experiments. The contexts for validation studies thus far have included:

- Expert evaluation of the content, construct and face validity of real psychological profiles compared with simulation-based profiles of learners.

- Experienced teachers create simulated students that mirror real students they are working with, and conduct parallel instructional and interactional interventions for comparison.

- Pre and post-tests of knowledge, skills and attitudes of people who are training to become teachers; these tests indicate impacts of the simulation on users.

- Treatment-comparison group impact differences. 
- Survey of users three years after treatment indicating higher retention in service compared to national averages in the U.S. This suggests that the simulation better prepares people to teach than other methods.

- Test-retest reliability of repeated measures.

- Internal reliability of measures regarding major constructs of experience versus confidence, resulting in a new measure of "pedagogical balance" in self-reports.

Additional replication and extension studies are needed. The model will need revision if empirical findings indicate gaps between learning theory and instructional practice that are unexplainable by, or missing in, the model's algorithms. Some future research findings are anticipated that will require an extension of the model either in its overall scope of defining learning as a physical-pre-conscious-cognitive complex (hubs) coevolving with an environment, or for further elaboration of key factors within the hubs (e.g. "decoding" as a sub-element of "reading" as a sub element of "language ability"), or for the introduction of new processing layers and linkages among the factors, some of which might be keyed to engage only in certain contexts or domains of performance.

\subsection{Testable Hypotheses}

The testable hypotheses (exploratory as well as confirmatory) within educationallyfocused studies of simSchool include a wide range of possibilities that span from individualized learning to classroom social processes and include the choices and behaviors of the teacher as a primary actor in a network of relationships, as well as the further validation of the COVE model instantiated in simSchool. For example, questions such as the following are a sampling of those that can be can be raised, for which data can automatically be collected by simSchool to provide visualizations and an analytic basis for insights into learning processes as well as some of the epistemological issues of teaching and learning:

1. Q: How does psychological differentiation among students impact group performance on academic tasks and on verbal interactions with a teacher? (The confirmatory hypothesis has been shown - psychological differentiation among students does impact academic performance and classroom interactions).

2. Q: How do teacher decisions about sequencing tasks differentially impact student learning? (The confirmatory hypothesis has been shown - teacher decisions do differentially impact student learning)

3. Q: How does the emotional stance of students impact their ability to learn?

4. Q: Are there linkages between physical, pre-conscious and cognitive factors that are empirically based and needed for accurate simulation of learning?

5. Q: Does a teacher have a preferred pattern of approaching students? Does that pattern differ by student, differ over time, and differ on other dimensions?

6. Q: How does the structure of an environment shape a student's emotional responses to teacher interactions and task requirements? 
7. Q: Is the COVE model of affective processes sufficient for certain purposes at its current level of description, and under what conditions are other descriptive levels needed?

The range of testable hypotheses will be increased by the addition of new parts of the simSchool model that are needed for classroom simulation; most particularly, social interactions among the students, and short and long term memory with specific domain contents. With these extensions of the modeling space, the testable questions will expand further into social-constructivist theories of learning.

\section{$7 \quad$ Summary}

This article presented a practical and operational framework for a cognitive, psychological, physical and environmental (COVE) model of learning, including a transient preconscious-processing layer in an AI agent's representation of learning in a serious game. The model organizes a hierarchy of cognitive (C), psychological (O) and physical (V) functioning of agents in an environment (E). The classroom-learning context of the simSchool application and its model of an AI student's reactions to academic tasks and teacher talk were utilized to discuss the theoretical foundations, design decisions, assumptions and implications of the model.

\section{References}

[1] L. Darling-Hammond, "Quality teaching: The critical key to learning," Principal, vol. 77, pp. 5-6, 1997.

[2] L. Darling-Hammond and P. Youngs, "Defining 'Highly Qualified Teachers': What Does 'Scientifically-Based Research' Actually Tell Us?," Educ. Res., vol. 31, no. 9, pp. 13-25, 2002.

[3] J. Rice, Teacher quality: Understanding the effectiveness of teacher attributes. Washington, DC: Economic Policy Institute, 2003.

[4] G. Girod, M. Girod, and J. Denton, "Lessons learned modeling 'connecting teaching and learning," in Games and simulations in online learning: Research \& development frameworks, D. Gibson, C. Aldrich, and M. Prensky, Eds. Hershey, PA: Idea Group, 2007, pp. 206 - 222.

[5] M. Girod and J. Girod, "Simulation and the need for quality practice in teacher preparation," American Association of Colleges for Teacher Education. 2006.

[6] P. Mishra and M. J. Koehler, "Technological Pedagogical Content Knowledge: A Framework for Teacher Knowledge," Teach. Coll. Rec., vol. 108, no. 6, pp. 1017-1054, 2006.

[7] C. A. Tomlinson, How to differentiate instruction in mixed-ability classrooms. Alexandria, Va.: Association for Supervision and Curriculum Development, 1995, p. iv, 80 p. 
[8] P. Grossman, "Research on pedagogical approaches in teacher education," in Studying teacher education, M. Cochran-Smith, Ed. Washington DC: American Educational Research Association, 2005, pp. 425-476.

[9] C. Aldrich, Simulations and the future of learning: an innovative (and perhaps revolutionary) approach to e-learning. San Francisco: John Wiley \& Sons, 2004.

[10] J. Foreman, J. Gee, J. Herz, R. Hinrichs, M. Prensky, and B. Sawyer, "Gamebased learning: How to delight and instruct in the 21 st century," Educ. Rev., vol. 39, no. 5, pp. 50-66, 2004.

[11] M. Prensky, Digital game-based learning. New York: McGraw-Hill, 2001.

[12] S. Brave and C. Hass, "Emotion in human-computer interaction," in The human-computer interaction handbook: Fundamentals, evolving technologies and emergine applications., J. Jacko and . Sears, Eds. Mahwah, N.J.: Lawrence Erlbaum Associates, Inc, 2003, pp. 81-96.

[13] M. Mayrath, J. Clarke-Midura, and D. Robinson, "Introduction to Technology-based assessments for 21st Centry skills," in Technology-based assessments for 21st Century skills: Theoretical and practical implications from modern research, M. Mayrath, J. Clarke-Midura, D. Robinson, and G. Schraw, Eds. Charlotte, NC: Information Age Publishers, 2012, p. 386.

[14] J. Clarke-Midura, J. Code, C. Dede, M. Mayrath, and N. Zap, "Thinking outside the bubble: Virtual performance assessments for measuring complex learning.," in Technology-based assessments for 21st Century skills: Theoretical and practical implications from modern research., Charlotte, NC: Information Age Publishers, 2012, pp. 125-148.

[15] R. Mislevy, L. Steinberg, and R. Almond, "Evidence-centered assessment design," Educational Testing Service, 1999.

[16] R. Christensen, T. Tyler-Wood, G. Knezek, and D. Gibson, "SimSchool: An online dynamic simulator for enhancing teacher preparation," Int. J. Learn. Technol., vol. 6, no. 2, pp. 201-220, 2011.

[17] G. Knezek and K. Vandersall, "simMentoring Results," simZine, vol. 2008. CurveShift, Stowe, p. 3, 2008.

[18] G. Knezek, P. Fisser, D. Gibson, R. Christensen, and T. Tyler-Wood, "SimSchool: Research outcomes from simulated classrooms," in Society for Information Technology \& Teacher Education International Conference 2012, 2012.

[19] L. Festinger, A theory of cognitive dissonance. Standford, CA: Standford University Press, 1957.

[20] J. Piaget, The equilibration of cognitive structures: The central problem of intellectual development. 1985.

[21] S. D’Mello and A. Graesser, "Modeling Cognitive-Affective Dynamics with Hidden Markov Models," in Annual Meeting of the Cognitive Science Society, 2010, pp. 2721-2726.

[22] R. McCrae and P. Costa, "Validation of the five-factor model of personality across instruments and observers.," J. Pers. Soc. Psychol., vol. 52, no. 1, pp. 81-90, 1987. 
[23] O. John and S. Srivastava, "The Big Five trait taxonomy: History, measurement, and theoretical perspectives.," in Handbook of personality: Theory and research, L. Pervin, Ed. New York: Guilford Press, 1999, pp. 102-138.

[24] B. M. Fridhandler, "Conceptual note on state, trait, and the state $\square$ "trait distinction.," Journal of Personality and Social Psychology, vol. 50. pp. 169174, 1986.

[25] W. Hofstee, B. de Raad, and L. Goldberg, "Integration of the Big Five and Circumplex approaches to trait structure," J. Pers. Soc. Psychol., vol. 63, no. 1, pp. 146-163, Jul. 1992.

[26] H. Eysenck and M. Eysenck, Personality and individual differences. New York: Plenum, 1985.

[27] L. S. Vygotsky, Mind in society: The development of higher psychological processes. 1978.

[28] A. Elliot, "Approach and avoidance motivation and achievement goals," Educ. Psychol. 32 1-19, vol. 34, pp. 149-169, 1999.

[29] H. Gardner, Multiple Intelligences: The theory in practice. New York: Basic Books, HarperCollins, 1993.

[30] G. Gay, "Culturally responsive teaching theory, research, and practice," Multicultural education series; Variation: Multicultural education series (New York, N.Y.). Teachers College Press, 2000.

[31] D. Gibson, "SimSchool-A complex systems framework for modeling teaching \& learning," in National Educational Computing Conference, Atlanta, GA, 2007.

[32] A. Ortony, G. Clore, and A. Collins, The cognitive structure of emotions. Cambridge, UK: Cambridge University Press, 1988.

[33] K. R. Scherer, "Appraisal theory. Handbook of cognition and emotion.," in Handbook of cognition and emotion, 1999, pp. 637-663.

[34] Merriam-Webster, "Merriam-Webster Online Dictionary," MerriamWebster Online Dictionary, vol. 2006. Merriam-Webster Online Dictionary, 2008.

[35] S. J. Cooper, "From Claude Bernard to Walter Cannon. Emergence of the concept of homeostasis.," Appetite, vol. 51, no. 3, pp. 419-427, 2008.

[36] L. Cosmides and J. Tooby, "Evolutionary psychology: A primer." University of California Santa Barbara, Santa Barbara, CA, 2007.

[37] L. Cosmides and J. Tooby, "Evolutionary Psychology and the Emotions," Handb. Emot., vol. 2, no. 1, pp. 91-115, 2000.

[38] D. Goleman, Emotional intelligence: Why it can matter more than IQ. New York: Bantam Books, 1995.

[39] J. Averill, "Together again: Emotions and intelligence reconciled," in The science of emotional intelligence. Knowns and unknowns., G. Matthews, M. Zeidner, and R. Roberts, Eds. New York: Oxford University Press, 2007, pp. 49-71.

[40] S. Budaev, "The dimensions of personality in humans and other animals: A comparative and evolutionary perspective.” Moscow, 2000. 
[41] V. Braitenberg, Vehicles, experiments in synthetic psychology. Cambridge, MA: MIT Press, 1984.

[42] R. Pfeifer and J. Bongard, How the body shapes the way we think: A new view of intelligence. Cambridge, MA: MIT Press, 2007.

[43] E. O. Wilson, "What is sociobiology?," Society, vol. 15, no. 6, pp. 10-14, 1978.

[44] B. Bloom, M. Engelhart, E. Furst, W. Hill, and D. Krathwohl, Taxonomy of educational objectives; The classification of educational goals. New York: David McKay, 1956.

[45] H. Gardner, Frames of mind: the theory of multiple intelligences. New York: Basic Books, 1983, p. xiii, 440 p.

[46] G. Edelman and G. Tononi, "Neural darwinism: The brain as a selectional system," in Nature's imagination: The frontiers of scientific vision, J. J. Cornwell, Ed. New York: Oxford University Press, 1995, pp. 148-160.

[47] K. McGrew, "Cattell-Horn-Carroll CHC (Gf-Gc) Theory: Past, Present \& Future,” vol. 2007, no. May 8. Institute for Applied Psychometrics, 2003.

[48] R. Cattell, Personality and motivation: Structure and measurement. New York: Harcourt, Brace \& World, 1957.

[49] J. Bruner, R. Oliver, and P. Greenfield, Studies in cognitive growth. New York: Wiley, 1966.

[50] E. Baum, What is thought? Cambridge, MA: MIT Press, 2004.

[51] R. Brooks, "A robust layered control system for a mobile robot," J. Robot. Autom., vol. 2, no. April, pp. 14-23, 1986.

[52] J. B. Carroll, "The three-stratrum theory of cognitive abilities," in Contemporary intellecual assessment: Theories, tests, and issues, D. Flanagan, J. Genshaft, and P. Harrison, Eds. New York: Guilford Press, 1996, pp. 122-130.

[53] K. McGrew and R. Woodcock, Technical manual. Itasca, IL: Riverside Publishing, 2001.

[54] K. LaBar and E. Phelps, "Arousal-mediated memory consolidation: Role of the medial temporal lobe in humans," Psychol. Sci., vol. 9, pp. 490-493, 1998.

[55] J. Hawkins and S. Blakeslee, On intelligence. New York: Henry Holt and Company, 2004.

[56] R. McCrae and P. Costa, "Toward a new generation of personality theories: Theoretical contexts for the five-factor model," in The five-factor model of personality: Theoretical perspectives, J. S. Wiggins, Ed. New York: Guilford, 1996, pp. 51-87.

[57] J. Russell, "Core affect and the psychological construction of emotion," Psychol. Rev., vol. 110, no. 1, pp. 145-172, 2003.

[58] R. Ewen, Personality: A topical approach. Mahweh, NJ: Erlbaum, 1998.

[59] J. Digman, "Personality structure: Emergence of the five-factor model," Annu. Rev. Psychol., vol. 41, pp. 417-440, 1990.

[60] R. M. Bagby, P. T. Costa, T. A. Widiger, A. G. Ryder, and M. Marshall, "DSM-IV personality disorders and the five-factor model of personality: A 
multi-method examination of domain- and facet-level predictions," Eur. J. Pers., vol. 19, pp. 307-324, 2005.

[61] G. Nestadt, P. T. Costa, F. C. Hsu, J. Samuels, O. J. Bienvenu, and W. W. Eaton, "The relationship between the five-factor model and latent Diagnostic and Statistical Manual of Mental Disorders, Fourth Edition personality disorder dimensions," Compr. Psychiatry, vol. 49, pp. 98-105, 2008.

[62] T. Chamorro-Premuzic and A. Furnham, "A possible model for understanding the personality-intelligence interface," Br. J. Psychol., vol. 95, pp. 249-264, 2004.

[63] S. Marsella and J. Gratch, "EMA: A process model of appraisal dynamics," Cogn. Syst. Res., vol. 10, no. 1, pp. 70-90, 2009.

[64] J. Broekens, "Modeling the experience of emotion," Int. J. Synth. Emot., vol. 1, pp. $1-17,2010$.

[65] M. Lahnstein, "The emotive episode is a composition of anticipatory and reactive evaluations," AISB Press, 2005.

[66] J. Berger, E. Cohen, and M. Zelditch Jr., "Status characteristics and expectation states," in Sociological theories in progress, vol. 1, J. Berger and M. Zelditch Jr., Eds. Boston: Houghton-Mifflin, 1966, pp. 29-46.

[67] W. Kalkhoff and S. Thye, "Expectation states theory and research: New observations from meta-analysis," Sociol. Methods Res., vol. 35, no. 2, pp. 219-249, 2006.

[68] J. Bransford, A. Brown, and R. Cocking, How people learn: Brain, mind, experience and school. Washington: DC: National Academy Press, 2000.

[69] R. Mcpherson, T. Tyler-wood, A. Ellison, and P. Peak, "Using a Computerized Classroom Simulation to Prepare Pre-Service Teachers," J. Technol. Teach. Educ., vol. 19, no. 1, pp. 93-110, 2011.

[70] O. Ellison, A. M., Tyler-Wood, T.L., Periathiruvadi, S., Sayler, M., Barrio, B., \& Lim, "Preservice teacher experiences with a computerized classroom simulation: A content analysis of discussion postings.," 2011.

[71] G. Knezek and R. Christensen, "Preservice educator learning in a simulated teaching environment," in Research Highlights in Technology and Teacher Education, vol. 1, no. 1, 2009, pp. 161-170.

[72] E. Reidel, “Teacher beliefs \& preparation survey,” Minneapolis, MN, 2000.

[73] R. DeVellis, Scale development. Newbury Park, NJ: Sage Publications, 1991.

[74] G. Knezek, R. Christensen, T. Tyler-Wood, P. Fisser, and D. Gibson, "SimSchool: Research outcomes from simulated classrooms," in Society for Information Technology \& Teacher Education International Conference 2012, 2012. 